\title{
Gastrointestinal stromal tumor (GIST) presenting with acute pancreatitis
}

A 58-year old woman presented with constant epigastric abdominal pain radiating to her back, of 1 week's duration. The patient had associated nausea, vomiting, anorexia, and weight loss. Physical examination revealed midepigastric tenderness without a palpable mass. Pertinent laboratory values included serum amylase of 410 units/L and lipase of 471 units/L.

Abdominal computed tomography (CT) scan revealed a 6 -cm cystic mass between the stomach and pancreas, as well as mild pancreatitis ( Fig. 1). Upper endoscopy showed extrinsic compression of the stomach along the lesser curvature, prohibiting passage of the scope to the pylorus. Endoscopic ultrasound revealed a $6 \times 6-\mathrm{cm}$ heterogeneous, well-rounded mass with calcifications and a calcified rim originating from the gastric mucosa, along with upstream dilation of the pancreatic duct to $1 \mathrm{~cm}$ ( Fig. 2 ). Fine-needle aspiration revealed spindle cells. During surgery, a well-rounded mass originating from the gastric antrum was found to have prolapsed into the second portion of the duodenum, obstructing the pancreatic duct. The patient underwent a partial resection of the anterior gastric wall. Final surgical pathology revealed diffuse c-kit positivity, confirming the mass was a gastrointestinal stromal tumor (GIST). Postoperatively, the patient's pancreatitis resolved, and she has not had a recurrence of her pancreatitis.

Although there are reports of duodenal GISTs mimicking pancreatic cancer, there are no known reports where acute pancreatitis (fulfilling two of three criteria) is secondary to a GIST [1]. In conclusion, we report a rare case of gastrointestinal stromal tumor presenting as acute pancreatitis, which, to our knowledge, is the first reported case in the literature.

Endoscopy_UCTN_Code_CCL_1AB_2AD_3AB

Competing interests: None
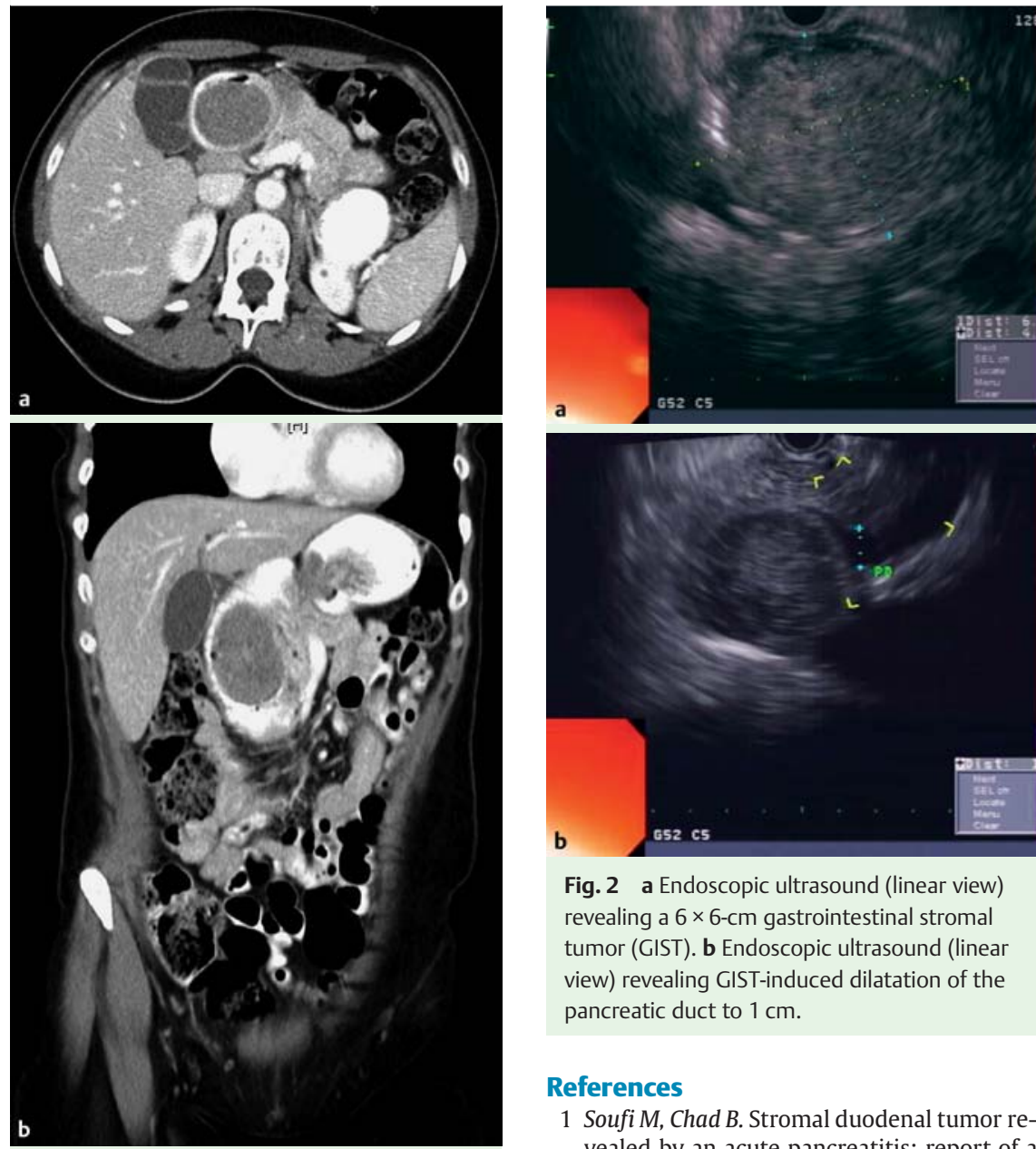

Fig. 1 a Gastrointestinal stromal tumor (GIST) obstructing the pancreatic head, with upstream dilation. b Prolapsed GIST protruding into the duodenum and obstructing the pancreatic duct.

\section{Senadhi ${ }^{1}$, D. Arora ${ }^{2}$, N. Jani ${ }^{1,3}$}

1 Johns Hopkins University/Sinai Hospital Program in Internal Medicine, Sinai Hospital, Baltimore, Maryland, USA

2 Elmhurst Hospital/Mount Sinai School of Medicine, New York, USA

3 Division of Gastroenterology, Greater Baltimore Medical Center (GBMC), Baltimore, Maryland, USA

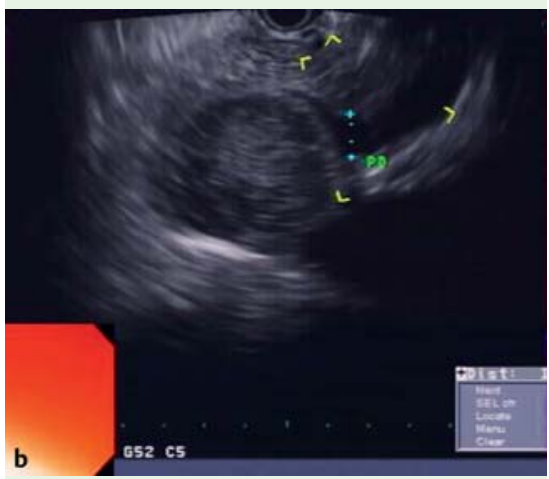

Fig. 2 a Endoscopic ultrasound (linear view) revealing a $6 \times 6-\mathrm{cm}$ gastrointestinal stromal tumor (GIST). b Endoscopic ultrasound (linear view) revealing GIST-induced dilatation of the pancreatic duct to $1 \mathrm{~cm}$.

\section{References}

1 Soufi M, Chad B. Stromal duodenal tumor revealed by an acute pancreatitis: report of a case. J Gastrointest Canc 2010; 41: 88-91

\section{Bibliography}

DOI $10.1055 / \mathrm{s}-0030-1256255$

Endoscopy 2011; 43: E76

(c) Georg Thieme Verlag KG Stuttgart · New York . ISSN 0013-726X

\section{Corresponding author}

\section{Senadhi}

Johns Hopkins University/Sinai Hospital Program in Internal Medicine

2606B Gage Court

Baltimore

Maryland 21209

USA

Fax: +1-678-623-5999

vsenadhi@hotmail.com 